\title{
Spatially Uniform ReliefF (SURF) for computationally-efficient filtering of gene-gene interactions Casey S Greene ${ }^{1}$, Nadia M Penrod ${ }^{1}$, Jeff Kiralis ${ }^{1}$ and Jason H Moore*1,2,3,4,5
}

Address: ${ }^{1}$ Department of Genetics, Norris Cotton Cancer Center, Dartmouth Medical School, Lebanon, NH, USA, ${ }^{2}$ Department of Community and Family Medicine, Dartmouth Medical School, Lebanon, NH, USA, ${ }^{3}$ Department of Computer Science, University of New Hampshire, Lebanon, NH, USA, ${ }^{4}$ Department of Computer Science, University of Vermont, Burlington, VT, USA and ${ }^{5}$ Translational Genomics Research Institute, Phoenix, AZ, USA

Email: Casey S Greene - casey.s.greene@dartmouth.edu; Nadia M Penrod - nadia.m.penrod@dartmouth.edu; Jeff Kiralis - jeff.kiralis@dartmouth.edu; Jason H Moore* - jason.h.moore@dartmouth.edu

* Corresponding author

Published: 22 September 2009

BioData Mining 2009, 2:5 doi:10.1 186/1756-038I-2-5

This article is available from: http://www.biodatamining.org/content/2/I/5

Central Ltd.

This is an Open Access article distributed under the terms of the Creative Commons Attribution License (http://creativecommons.org/licenses/by/2.0), which permits unrestricted use, distribution, and reproduction in any medium, provided the original work is properly cited.

\begin{abstract}
Background: Genome-wide association studies are becoming the de facto standard in the genetic analysis of common human diseases. Given the complexity and robustness of biological networks such diseases are unlikely to be the result of single points of failure but instead likely arise from the joint failure of two or more interacting components. The hope in genome-wide screens is that these points of failure can be linked to single nucleotide polymorphisms (SNPs) which confer disease susceptibility. Detecting interacting variants that lead to disease in the absence of singlegene effects is difficult however, and methods to exhaustively analyze sets of these variants for interactions are combinatorial in nature thus making them computationally infeasible. Efficient algorithms which can detect interacting SNPs are needed. ReliefF is one such promising algorithm, although it has low success rate for noisy datasets when the interaction effect is small. Relieff has been paired with an iterative approach, Tuned ReliefF (TuRF), which improves the estimation of weights in noisy data but does not fundamentally change the underlying ReliefF algorithm. To improve the sensitivity of studies using these methods to detect small effects we introduce Spatially Uniform ReliefF (SURF).

Results: SURF's ability to detect interactions in this domain is significantly greater than that of ReliefF. Similarly SURF, in combination with the TuRF strategy significantly outperforms TuRF alone for SNP selection under an epistasis model. It is important to note that this success rate increase does not require an increase in algorithmic complexity and allows for increased success rate, even with the removal of a nuisance parameter from the algorithm.

Conclusion: Researchers performing genetic association studies and aiming to discover gene-gene interactions associated with increased disease susceptibility should use SURF in place of ReliefF. For instance, SURF should be used instead of ReliefF to filter a dataset before an exhaustive MDR analysis. This change increases the ability of a study to detect gene-gene interactions. The SURF algorithm is implemented in the open source Multifactor Dimensionality Reduction (MDR) software package available from http://www.epistasis.org.
\end{abstract}




\section{Background}

Technological advances are rapidly improving geneticists ability to measure variation between individuals. Because of these advances, the genome-wide association study is now a common approach to detecting genetic factors which influence individual susceptibility to common human diseases. Genome-wide association studies targeting common variants which, alone, influence susceptibility have produced mixed results [1-5]. As currently performed, these studies ignore complex interactions between variants that may lead to disease susceptibility. These are often ignored because methods to detect these interactions are computationally infeasible or provide insufficient sensitivity.

Epistasis is a term literally meaning "resting upon" which refers to the situation where interacting genes, as opposed to a single gene, influence a trait. Because of the complex architecture of biological networks, epistasis is likely to be fundamental to an individual's disease risk for common human diseases [6]. This, combined with the knowledge that single-locus results have not frequently replicated for common human diseases $[7,8]$, indicates that methods to detect and characterize epistasis are likely to be critical to understanding the genetic basis of common human disease.

Detecting and characterizing epistatic interactions in datasets containing large numbers of SNPs is challenging. It requires examining the effect of SNPs not just in isolation, but also in concert with other SNPs. In a dataset with one million SNPs, a number typically provided by high throughput technologies, there are about $5 \times 10^{11}$ pairwise combinations of SNPs. For three-way combinations, the number is $1.7 \times 10^{17}$. For higher order interactions the number of combinations is astronomical. Combinatorial methods which evaluate each such combination are not feasible [9].

Efficient algorithms for identifying sets of SNPs likely to contain predictive models for disease susceptibility are therefore needed. Methods of filtering SNPs are one possibility. These first rank the attributes by some criterion. Then either the top $K$ SNPs or all SNPs above some threshold $T$ are selected. The SNPs within this set can then be analyzed for interactions using combinatorial methods. Stochastic search wrappers are another possibility. These wrappers are probabilistic methods which retain the ability to consider all attributes and have the potential to use information learned early in the search to direct future exploration. Relief algorithms are nearest neighbor based approaches to detecting attributes relevant for some outcome. Relief algorithms are attractive for use in genetic association studies using either filters or wrappers because the computation time required increases linearly with the number of SNPs and quadratically with the number of individuals. Importantly, these algorithms are able to detect attributes associated with disease through interactions or independent main effects, although they do not provide a model for the effect [10]. Instead, information gleaned from these methods can be used as input into other approaches. Stochastic search approaches such as genetic programming [11-13] and ant colony optimization [14] can successfully develop models in this domain when information from the Relief family of algorithms is used to assist the search, although they fail to detect purely epistatic associations without this additional information [12]. Motsinger et al. [15] have shown that patterns of correlation between SNPs can make the problem easier to solve in the absence of expert knowledge, although here we specifically examine uncorrelated SNPs. Moore et al. briefly discuss both filter and wrapper options as part of an overall epistasis analysis strategy for human disease susceptibility [16] and Greene et al. [17] provide a theoretical analysis of both approaches. For the situation where there is a single source of expert knowledge, the filter approach is most appropriate [17]. In this situation we are considering the success rate of individual Relief methods, each of which is a single source which meets these assumptions up to a good approximation according to the appendix (Additional file 1). For this reason we test the ability of these methods to successfully filter a dataset retaining SNPs with an epistatic interaction associated with disease susceptibility.

Numerous variants of Relief have been developed. When applied to genetic association study data these methods use genetically similar individuals or, equivalently, nearest neighbors to adjust weights which are assigned to each SNP. The nearest neighbor is the nearest individual in the dataset to the current individual calculated across all SNPs. While Relief uses, for each individual, a single nearest neighbor in each class, ReliefF, a variant of Relief, uses multiple nearest neighbors, and thus is more robust when the dataset contains noise [18]. Moore and White developed a Tuned ReliefF (TuRF) approach for human genetics [19]. This approach, though requiring more computer time, further improves the performance when the data contain a large number of non-relevant SNPs in addition to a small number of relevant SNPs. TuRF achieves this by iterating a ReliefF algorithm and, with each iteration, deleting SNPs with the lowest ReliefF weights, i.e. those thought to be least predictive [19]. SNPs are assigned a weight based on their normalized weights when removed. This iterative approach improves the overall ranking of disease associated SNPs because noisy SNPs are most often removed. This means that the re-estimation can more accurately evaluate the relevance of the remaining SNPs. 
Here we present a new version of Relief, called Spatially Uniform ReliefF or, briefly, SURF. It detects epistatic interactions with a significantly higher success rate than the Relief variant widely used for machine learning, ReliefF. Iterated SURF, called SURF \& TuRF, has a significantly higher success rate than TuRF. For each individual SURF, like ReliefF, adjusts weights of all the SNPs by using certain neighbors of the individual. While Relieff uses a fixed number of nearest neighbors, SURF uses all neighbors within a fixed distance of the individual. This distance may be thought of as a similarity threshold. Thus SURF uses precisely those neighbors more similar than this threshold. Relieff, on the other hand, may use either fewer or more neighbors, thereby possibly neglecting informative individuals or including uninformative ones. Furthermore, similarity thresholds which give greater success rate than ReliefF can be estimated from the data while distances are pre-computed, thus removing a nuisance parameter from the algorithm (see $\$ 2$ in the appendix). SURF also does not increase the complexity of the algorithm, so the scaling is still linear with respect to the number of SNPs and quadratic with respect to the number of individuals.

\section{Relief and Spatially Uniform ReliefF (SURF)}

All Relief algorithms attach a weight to each SNP. The higher the weight of a SNP, the more likely it is predictive of disease status. Genetically similar individuals are used to adjust these SNP weights. We define the distance between two individuals as the number of their SNPs with differing genotypes. With this distance metric, nearest neighbors share genotypes at the greatest number of SNPs, and so are genetically most similar.

Relief algorithms are based on the assumption that those SNPs of nearby individuals which have different states (i.e. differing genotypes) are either most or least predictive of disease status. Relief algorithms adjust the weights of these SNPs-upward if the two individuals have different disease status, and downward by the same amount if they have the same status. More precisely, the original Relief algorithm adjusts, for each individual $I_{i}$, the SNP weights using $I_{i}$ 's nearest hit (the individual which is closest to $I_{i}$ and in the same class as $I_{i}$ ) and $I_{i}$ 's nearest miss (the individual which is closest to $I_{i}$ and in the other class from $I_{i}$ ). In the case of SURF, for each individual $I_{i}$, this adjustment is done using each hit and miss within a fixed threshold distance $T$ of $I_{i}$. Figure 1 shows graphically how neighbors are selected with each Relief algorithm.

Relief is able to detect epistatic SNPs, even when no single SNP has an effect. We outline how it does this for epistatic pairs. More detail is in the appendix (Additional file 1). All of the penetrance functions used in this work are available in Additional file 2. We begin with a discussion of epistatic pairs. Consider the penetrance function for the

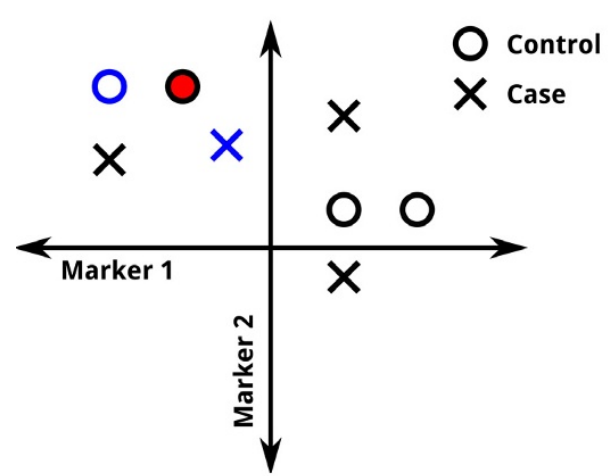

A) Relief

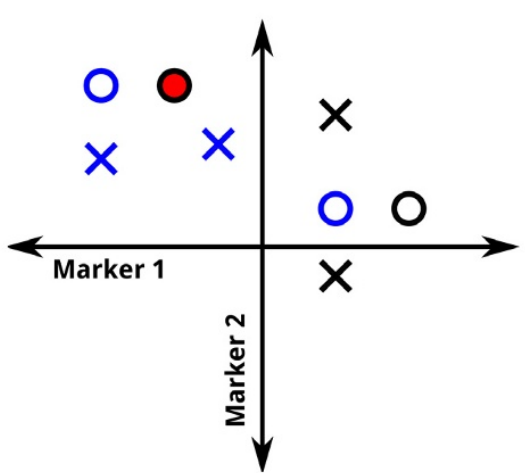

B) ReliefF

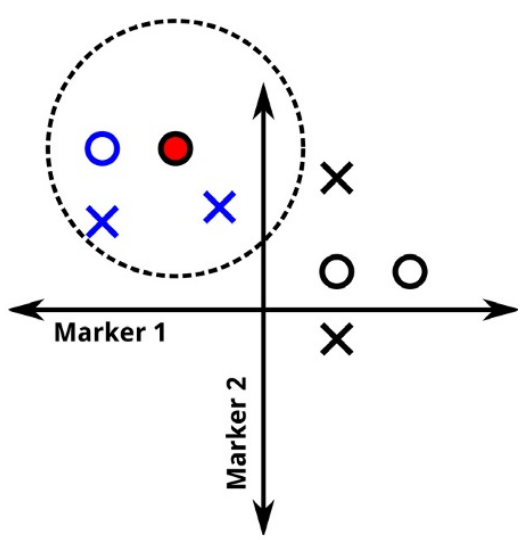

C) SURF

\section{Figure I}

How Relief, ReliefF and SURF select neighbors. Each panel in this figure shows the genotypes at two markers for a dataset of cases and controls. For the purpose of this example only these two markers will be considered and both are continuous. When analyzing real data, the process of selecting neighbors is the same, however, but there will be thousands of discrete valued markers (SNPs) each of which would be represented by one of thousands of dimensions. The individual for whom neighbors are being found is shown by the filled red circle. The neighbors that each approach uses for weighting are highlighted in blue. Parts A, B, and C represent how Relief, ReliefF and SURF would select neighbors to be used in weighting. Relief selects the nearest individual of the same class (blue circle) and the nearest individual of the other class (blue cross). ReliefF selects some user specified number of individuals (two in this example) to be used for weighting. SURF, instead of using a fixed number of neighbors, uses all individuals within a distance threshold. The dotted line shows a hypothetical distance threshold. 
epistatic pair of SNPs shown in Table 1. If an individual has genotype $\mathrm{AA}$ and the genotype of $\mathrm{SNP}_{2}$ is unknown, then the probability the individual is sick is

$$
.36 \cdot .469+.48 \cdot .337+.16 \cdot .339=.3848
$$

The individual has the same probability of being sick if he has genotype either Aa or aa, provided again that the genotype of $\mathrm{SNP}_{2}$ is unknown. Similarly, if his genotype is either $\mathrm{BB}, \mathrm{bB}$ or bb with $\mathrm{SNP}_{1}$ 's genotypes unknown, the probability he is sick is again .3849. The point is that no single SNP has an effect on disease susceptibility. Only the relevant pair does.

Now we discuss how Relief detects epistatic pairs. Given an individual $I_{i}$, we define the set $M_{k \Delta}$ to consist of those misses with exactly $k$ of their two relevant SNPs in a different state from those of individual $I_{i}$. In the case of two relevant SNPs, $k=0,1$ or 2 . Note that the miss nearest $I_{i}$ is in exactly one of the three sets $M_{0 \Delta^{\prime}} M_{1 \Delta}$ or $M_{2 \Delta}$. Indeed, these partition the set of all misses. The sizes of the sets $M_{k \Delta}$ can be determined (as in $\$ 1$ of the appendix) from the penetrance function which governs the relationship between genotype and phenotype. As an example, with a sample size of 1600 and the penetrance function shown in Table 1 the sizes of these sets are

$$
\left|M_{0 \Delta}\right|=110, \quad\left|M_{1 \Delta}\right|=397 \text { and }\left|M_{2 \Delta}\right|=293 \text {. }
$$

For the analogous sets involving hits we have

$$
\left|H_{0 \Delta}\right|=130, \quad\left|H_{1 \Delta}\right|=357 \text { and }\left|H_{2 \Delta}\right|=313 \text {. }
$$

These are actually expected numbers rounded to the nearest integer. Since $\left|M_{1 \Delta}\right|>\left|M_{2 \Delta}\right|$, the contribution of the irrelevant SNPs to the distance from $I_{i}$ to its nearest point in $M_{1 \Delta}$ tends to be less than that to its nearest point in $M_{2 \Delta}$. The two relevant SNPs contribute one to the distance from $I_{i}$ to every point of $M_{1 \Delta}$. For points in $M_{2 \Delta}$, the contribution to this distance is two, which makes points in $M_{2 \Delta}$ farther by one from $I_{i}$, on average, than points in $M_{1 \Delta}$. Since the states of the relevant and irrelevant SNPs are inde-

Table I: Penetrance values for an example epistasis model with a heritability of 0.1 .

\begin{tabular}{lcccc}
\hline & & \multicolumn{3}{c}{ SNP } \\
\cline { 3 - 5 } & & AA (0.36) & Aa (0.48) & aa (0.16) \\
\hline SNP 2 & BB (0.36) & 0.469 & 0.198 & 0.754 \\
\cline { 2 - 5 } & Bb (0.48) & 0.337 & 0.502 & 0.141 \\
\cline { 2 - 5 } & bb (0.16) & 0.339 & 0.453 & 0.285
\end{tabular}

pendent, it follows that the nearest miss is more likely to be in $M_{1 \Delta}$ than $M_{2 \Delta}$. To be precise for the example in table 1 the probability, according to equation (10) of the appendix, that the closest miss is in $M_{1 \Delta}$ is

$$
P_{C M}(1 \Delta) \approx .516
$$

while the probability it is in $M_{2 \Delta}$ is

$$
P_{C M}(2 \Delta) \approx .309 \text {. }
$$

We mention that the probability it is in $M_{0 \Delta}$ is

$$
P_{C M}(0 \Delta) \approx .175
$$

but do not use this since Relief adjusts weights only for SNPs where pairs of individuals have differing genotypes. The analogous probabilities for hits are

$P_{C H}(1 \Delta) \approx .463, \quad P_{C H}(2 \Delta) \approx .330$, and $P_{C H}(0 \Delta) \approx .207$.

If the nearest miss is in $M_{2 \Delta^{\prime}}$ then the Relief score of both relevant SNPs is increased by one. If it is in $M_{1 \Delta}$, there is a $50 \%$ chance that the score of the first relevant SNP is increased by one. Thus the expected contribution due to misses of individual $I_{i}$ to the score of a relevant SNP is

$$
\frac{1}{2} P_{C M}(1 \Delta)+P_{C M}(2 \Delta)
$$

Using the same notation for hits, except with $\mathrm{H}$ in place of $\mathrm{M}$, an analogous discussion gives

$$
-\left(\frac{1}{2} P_{C H}(1 \Delta)+P_{C H}(2 \Delta)\right)
$$

as the expected contribution dues to hits of individual $I_{i}$ to the score of a relevant SNP. Thus the expected contribution of individual $I_{i}$ to the score of a relevant SNP is

$$
\frac{1}{2}\left(P_{C M}(1 \Delta)+P_{C H}(1 \Delta)\right)+P_{C M}(2 \Delta)-P_{C H}(2 \Delta)
$$

The value of this for the example we have been considering is .005. The expected contribution of individual $I_{i}$ to the score of an irrelevant SNP is 0. This indicates why Relief tends to assign higher scores to relevant SNPs than to irrelevant ones.

The analysis of SURF, though mathematically easier, is more subtle. Again, let $I_{i}$ be a random, but fixed, individual. Then, as before, each miss within the threshold distance $T$ of $I_{i}$ is in one of the three sets $M_{0 \Delta}, M_{1 \Delta}$ or $M_{2 \Delta}$. For $k=0,1$ and 2 , let $T M_{k}$ be the subset of $M_{k \Delta}$ consisting of those individuals within distance $T$ of $I_{i}$. Using analogous 
notation for hits with $H$ in place of $M$, the mean contribution of individual $I_{i}$ to the SURF score of a relevant SNP is

$$
\begin{aligned}
S_{i}^{R} & =\frac{1}{2}\left(\left|T M_{1}\right|-\left|T H_{1}\right|\right)+\left(\left|T M_{2}\right|-\left|T H_{2}\right|\right) \\
& =\frac{1}{2}\left(\left|T M_{1}\right|-\left|T H_{1}\right|\right)-\left(\left|T M_{2}\right|-\left|T M_{2}\right|\right) .
\end{aligned}
$$

The $\frac{1}{2}$ is here since each individual in $T M_{1}$ and $T H_{1}$ changes the score of a relevant SNP by $\frac{1}{2}$, on the average.

Returning now to the example model, specifically expressions (1) and (2), we see that $\left|M_{2 \Delta}\right|-\left|H_{2 \Delta}\right|<0$.

Thus, on average, $\left|T M_{2}\right|-\left|T H_{2}\right|<0$; however two factors make $S_{i}^{R}>0$. Namely

$$
\left|M_{1 \Delta}\right|-\left|H_{1 \Delta}\right|=40>\left|H_{2 \Delta}\right|-\left|M_{2 \Delta}\right|=20
$$

making

$$
\left|T M_{1}\right|-\left|T H_{1}\right|>\left|T H_{2}\right|-\left|T M_{2}\right| .
$$

Also, elements of $M_{1 \Delta}$ and $H_{1 \Delta}$ are, on average, one closer to $I_{i}$ than elements of $M_{2 \Delta}$ and $H_{2 \Delta}$. Together these make

$$
\frac{1}{2}\left(\left|T M_{1}\right|-\left|T H_{1}\right|\right)>\left|T H_{2}\right|-\left|T M_{2}\right|
$$

and, consequently, $S_{i}^{R}>0$, on average. For the example penetrance function, equation (3) of the appendix gives $S_{i}^{R}=.519$; however, this SURF score cannot be reasonably compared to the analogous Relief score of .005 without a discussion of the variances of these scores. We do this in the appendix, and also indicate in $\$ 5$ why SURF outperforms ReliefF using 10 nearest neighbors.

The scores $S_{i}^{R}$ depend on the value of the distance threshold $T$. In our simulations, we have chosen $T$ to maximize the $S_{i}^{R}$. The final score of a relevant SNP is the sum of the $S_{i}^{R}$ values for each individual.

Because of the way the variance of this sum varies with $T$, slightly smaller values of $T$ are probably optimal. This is discussed at the end of $\$ 2$ of the appendix.

\section{Results and Discussion}

Our results suggest that the SURF approaches provide a more successful method for the detection of gene-gene interactions in these data. Figure 2 shows both success rate and significance test results for a single sample size and heritability (1600 and 0.1 respectively). These results indicate that the success rates of the SURF approaches (SURF and SURF \& TuRF) are greater than their corresponding ReliefF approaches (ReliefF and TuRF). Furthermore the step plots show that this difference is highly significant except for the $99^{\text {th }}$ percentile comparison of ReliefF and SURF. Neither of the non-iterative approaches is highly effective for filtering to the $99^{\text {th }}$ percentile for this heritability and sample size, so as a stringent filter the iterative approaches are most useful.

Our complete results, shown in figure 3, show that the new SURF algorithm, outperforms ReliefF. Furthermore we see that this increase in success rate is not redundant with the tuned approaches, as both of these, TuRF and SURF \& TuRF, which iteratively remove attributes with low quality estimates, are much better than the standard Relief and SURF approaches at selecting a small subset which contains the functional attributes. Here we see that these approaches significantly outperform ReliefF and SURF when the task is to filter the dataset to the $99^{\text {th }}$ or $95^{\text {th }}$ percentiles of SNPs. Finally we find that SURF \& TuRF outperforms TuRF alone achieving a much greater success rate, particularly at moderate heritabilities. We find that these differences are statistically significant. The success rate when SURF is used, particularly with larger sample sizes, is consistently significantly greater than the success rate when the standard method, ReliefF is used (see Additional files 3, 4, 5) for both the "tuned" and non-iterative approaches. Additionally the success rates of these "tuned" algorithms to include the proper SNP in the $99^{\text {th }}$ and $95^{\text {th }}$ percentiles are consistently significantly better than the success rates of the non-tuned approaches (see figure 2 and Additional files 3, 4, 5).

Methods which increase success rate without an increase in computational complexity or sample size are extremely desirable for genome-wide association studies. By developing improved methods for detecting epistasis we greatly expand our ability to characterize interactions in large datasets. Moore argues that when people use sensitive methods to detect epistasis, they are frequently able to find examples of it [20]. Algorithms which both detect and characterize epistasis in the absence of main effects are of combinatorial complexity for the number of SNPs. The SURF algorithm we introduce to detect disease associated interacting SNPs is, like ReliefF, of linear complexity for the number of SNPs. Moreover, its success rate for epistasis analysis is higher than ReliefF's. One caveat is that Relief methods such as SURF, though useful for detecting interacting SNPs, neither identify specific interacting pairs nor develop a model. Because SURF \& TuRF is able to detect interacting genetic variants which are pre- 

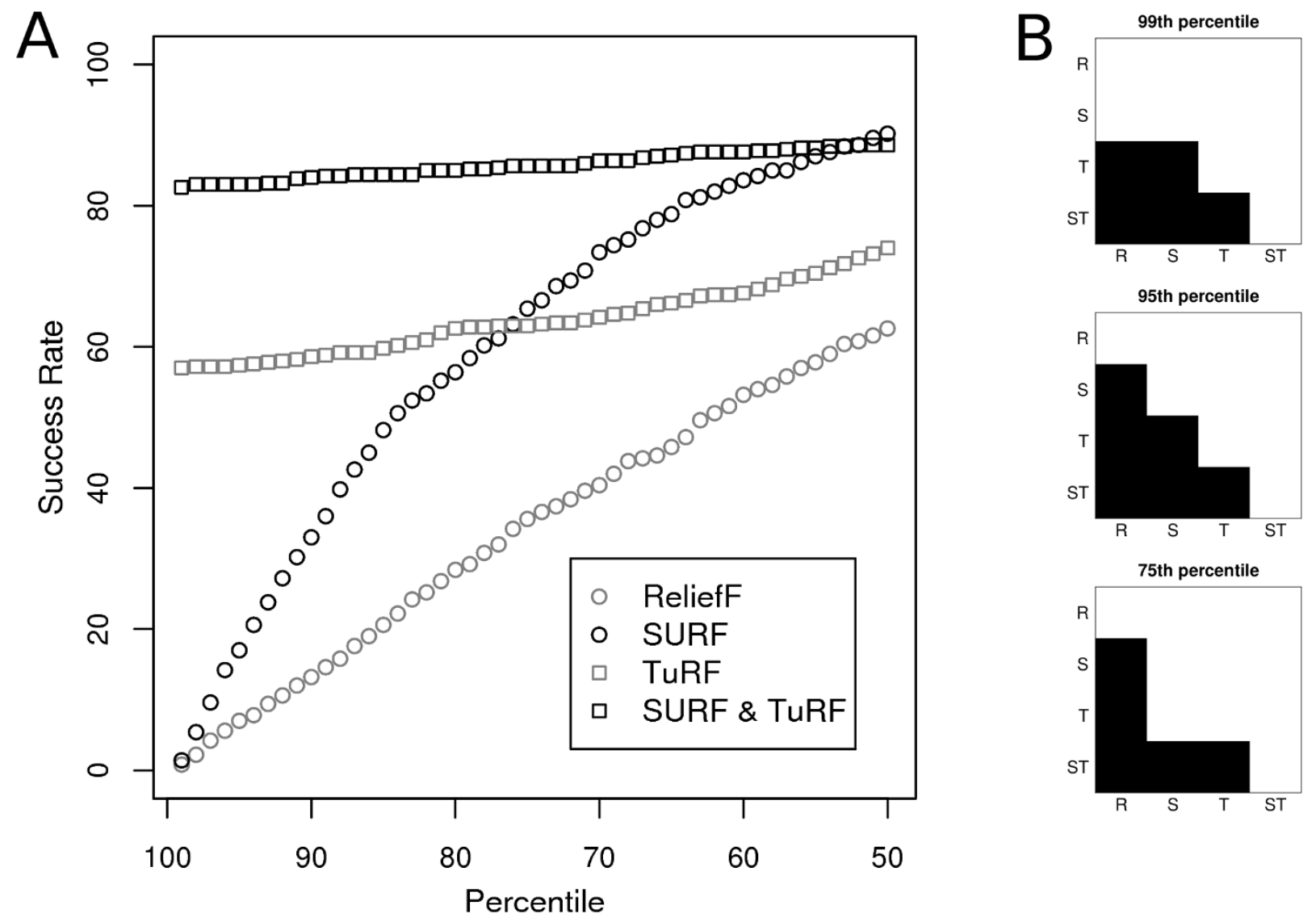

\section{Figure 2}

Example Success Rate and Significance of Differences. Part A shows the detailed success rate analysis results for a single heritability (0.I) and sample size (1600). The success rate to filter both relevant SNPs into percentiles from the 99 th to $50^{\text {th }}$ is shown. The $99^{\text {th }}$ percentile corresponds to the top 10 SNPs by the assigned weights in these datasets which contain 1000 SNPs. In part B pairwise comparisons are made between each pair of methods at the $99^{\text {th }}, 95^{\text {th }}$, and $75^{\text {th }}$ percentiles. ReliefF, SURF, TuRF, and SURF\&TuRF are labeled R, S, T, and ST respectively. Significance is illustrated with levels of grey (i.e. light grey indicates $0.0 \mathrm{I}<p \leq 0.05$, dark grey indicates $0.00 \mathrm{I}<p \leq 0.0 \mathrm{I}$, and black indicates $p \leq 0.00 \mathrm{I}$ ). As an example, at the 99 th $p e r c e n-$ tile the blank square at the intersection of $\mathrm{R}$ and $\mathrm{S}$ indicates that the difference between ReliefF and SURF was not significant. On the other hand the black square at the intersection of S and ST indicates that the difference between the success rates of SURF and SURF\&TuRF at that percentile was highly significant.

dictive of human health, weights from this algorithm can be used to filter a dataset before traditional combinatorial approaches are used to characterize the interaction. McKinney et al. have previously integrated ReliefF [21] and TuRF [22] with other information sources using an evaporative cooling technique to direct genetic association analyses. Direct replacement of ReliefF by SURF \& TuRF may improve the sensitivity of these frameworks to detect and characterize interactions.

SURF \& TuRF's greatly increased success rate to detect epistasis improves our ability to detect variants leading to disease risk in the absence of main effects. This new distance based approach may also be extensible to biological and biomedical data beyond case-control genetic association studies. While ReliefF, which SURF \& TuRF builds on, is usable for these discrete endpoints and attribute values, other modifications to ReliefF have extended this machine learning method to other data types. With Regression ReliefF (RReliefF), ReliefF is broadened to handle continuous attributes and endpoints [23,24]. Future work should examine whether the new distance based approach used for SURF \& TuRF also improves these methods. If using a distance threshold also improves RReliefF methods, the sensitive SURF approach can be applied to continuous gene expression data or to detecting variants predictive of continuous endpoints. With future work it may also be possible to combine continu- 

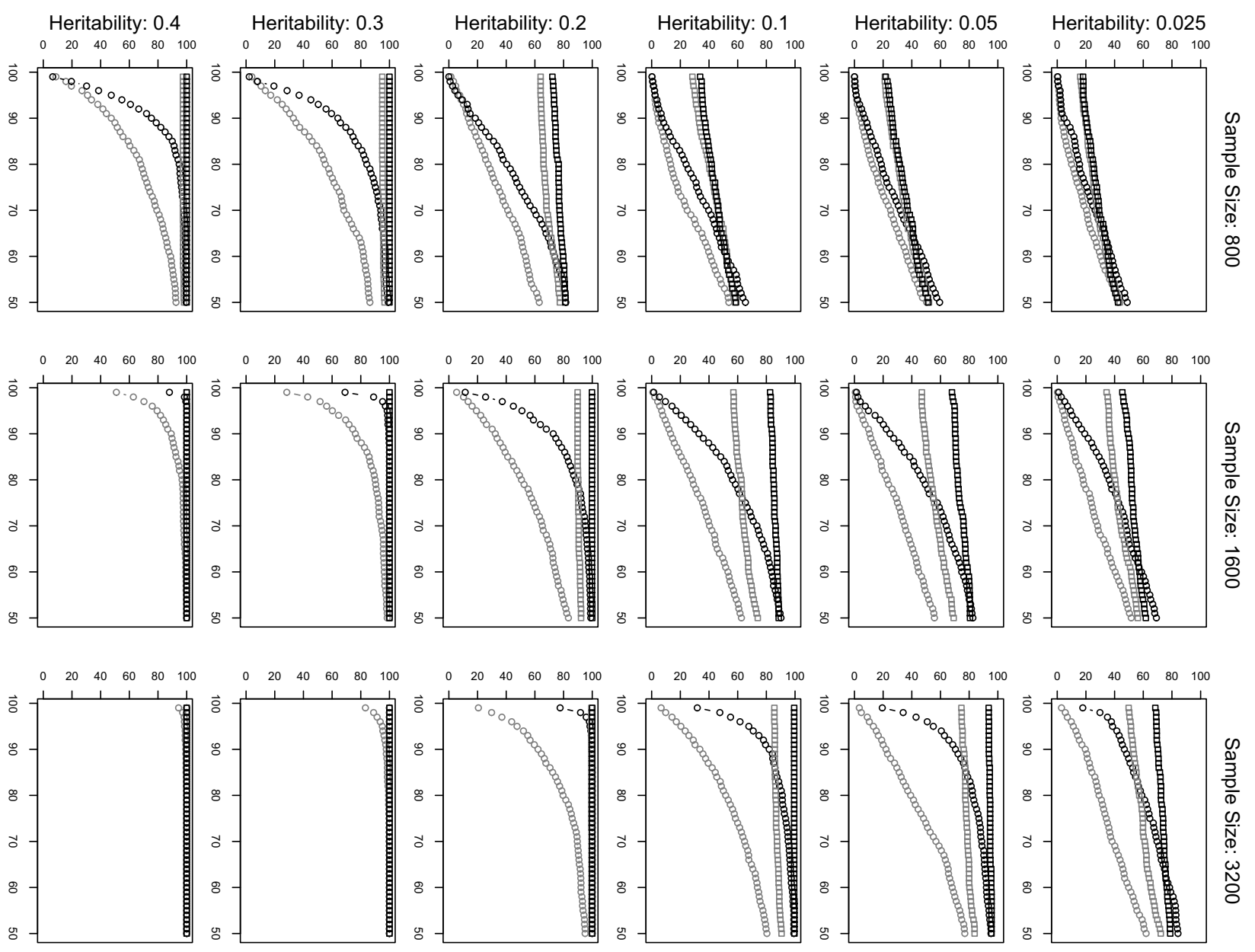

\section{Figure 3}

Success Rate Analysis. This is a summary of success rate as shown in figure 2 across a wide range of sample sizes and heritabilities. Within each heritability the success rates for all five genetic models for that heritability are averaged. The $x$-axis for each plot corresponds to the percentiles as in figure 2. Across these situations, SuRF alone performs as well as TuRF when filtering to the $75^{\text {th }}$ percentile of SNPs. SURF outperforms ReliefF, the tuned approaches outperform the non-tuned approaches when using a more stringent filter (i.e. 99th and $95^{\text {th }}$ percentiles), and SURF \& TuRF outperforms TuRF with ReliefF.

ous and discrete attributes, to provide a method capable of examining gene-gene, gene-environment, and environment-environment interactions in a common framework.

\section{Conclusion}

Now that it is technically and economically feasible to measure large numbers of DNA sequence variations in human genetics, the bioinformatics challenge is to identify and improve methods for detecting variants which are predictive of disease risk. This is particularly challenging when the task is to identify polymorphisms which have little or no independent effect. The Relief family of algorithms provides one potential solution for SNP selection, and SURF \& TuRF is a novel within this family which effectively detects epistasis. By developing sensitive and computationally efficient methods capable of detecting epistasis, it becomes more practical to probe datasets for these interactions. Highly sensitive methods will allow researchers to better understand the impact of epistasis on human health. Both SURF and SURF \& TuRF have been included as filtering methods in the user friendly open 
source Multifactor Dimensionality Reduction (MDR) software package.

\section{Methods}

As discussed SURF weights can be used for genetic analysis in either filters or probabilistic wrappers. Here we consider the simpler filter approach. Specifically we analyze SURF's ability to filter a dataset to the $99^{\text {th }}, 95^{\text {th }}$ and $75^{\text {th }}$ percentiles of SNPs without removing those SNPs with an interaction effect predictive of disease susceptibility. Relieff has previously been used in the genetic analysis of complex diseases in this fashion [25].

The goal of our simulation study is to generate artificial datasets with high concept difficulty to evaluate SURF in the domain of human genetics. We first develop 30 different penetrance functions (i.e. genetic models) which determine the relationship between genotype and phenotype in our simulated data. These functions determine the probability that an individual has disease given his or her genotype. This probability depends only on the genotypes of the two interacting SNPs, not on the genotype of any one SNP. The 30 penetrance functions include groups of five with heritabilities of $0.025,0.05,0.1,0.2,0.3$, or 0.4 . These heritabilities range from very small to large genetic effect sizes. Each functional SNP has two alleles with frequencies of 0.4 and 0.6. These models are included in Additional file 2. Each of the models is used to generate 100 replicate datasets with sample sizes of 800,1600 , and 3200. Each dataset consists of an equal number of case (diseased) and control (disease free) subjects. Each pair of functional SNPs is added to a set of 998 irrelevant SNPs for a total of 1000 attributes. A total of 9,000 datasets are generated and analyzed.

We test each method with the following parameters. All four methods can use some or all of the dataset when performing weight estimations. Here we use the entire dataset, as this is similar to what is performed in practice where the number of individuals is often more limiting than the computational costs. ReliefF and TuRF require a number of neighbors. Here we use 10, as suggested by Robnik-Sikonja and Kononenko [24] in a comprehensive analysis. SURF requires a distance threshold. Our theoretical analysis in $\$ 2$ of the appendix (Additional file 1) suggests that the mean distance between all pairs of individuals in the dataset and across all attributes can be used and thus we use this distance in this situation. By using the mean distance as calculated from the data, we remove this nuisance parameter from the algorithm. Both SURF \& TuRF and TURF remove a number of SNPs at each iteration before re-estimating the weights of the remaining SNPs. Here we remove 25 SNPs at each iteration ( $2.5 \%$ of the dataset).
Here, because we are interested in interactions, we consider the success rate to be the number of times that both relevant SNPs are scored above a given threshold. We set this stricter standard here because further analysis steps can not succeed of both relevant parts of the interaction are not discovered. To estimate the success rate, we use 100 datasets for each of the 30 models. Specifically, the percentage of datasets for which a method ranks the two relevant SNPs above the $N^{\text {th }}$ percentile of all SNPs is the estimate of the method's success rate. We apply Fisher's exact test to assess the significance of differences between the success rates of the tested methods at these thresholds. These percentiles represent the situation where each method is used to filter a large dataset with 1000 SNPs to a smaller dataset of 10, 50, and 250 SNPs respectively. Fisher's exact test is a significance test appropriate for categorical count data [26]. The resulting $p$-value for this test can be interpreted as the likelihood of seeing a difference of the size observed among success rates when the methods do not differ. We consider results statistically significant when $p \leq 0.05$. Additionally, we graphically show results for filtering to each percentile from the $99^{\text {th }}$ to the $50^{\text {th }}$.

\section{Competing interests}

The authors declare that they have no competing interests.

\section{Authors' contributions}

CSG and JK developed SURF. CSG, NMP and JHM designed and performed the experiments. CSG, NMP, JK and JHM prepared the manuscript. All authors have read and approved the final manuscript.

\section{Additional material}

\section{Additional file 1}

Appendix. This is an appendix to accompany the manuscript that includes additional theoretical analysis of the Relief algorithms discussed in the manuscript.

Click here for file

[http://www.biomedcentral.com/content/supplementary/17560381-2-5-S1.pdf]

\section{Additional file 2}

Epistasis models. These are the epistasis models used in our data simulation.

Click here for file

[http://www.biomedcentral.com/content/supplementary/17560381-2-5-S2.pdf] 


\section{Additional file 3}

Significance of differences with a sample size of 800 . This is a plot showing the significance of statistical results for the situation where there are 400 cases and 400 control individuals. These plots follow the example shown in Figure 2. Pairwise comparisons are made between each pair of methods at the $99^{\text {th }}, 95^{\text {th }}$, and $75^{\text {th }}$ percentiles. ReliefF, SURF, TuRF, and SURF \& TuRF are labeled $R, S, T$, and ST respectively. Significance is illustrated with levels of grey (i.e. light grey indicates $0.01<\mathrm{p} \leq 0.05$, dark grey indicates $0.001<\mathrm{p} \leq 0.01$, and black indicates $\mathrm{p} \leq 0.001$ ). Click here for file

[http://www.biomedcentral.com/content/supplementary/17560381-2-5-S3.pdf]

\section{Additional file 4}

Significance of differences with a sample size of 1600. This is a plot showing the significance of statistical results for the situation where there are 800 cases and 800 control individuals. These plots follow the example shown in Figure 2. Pairwise comparisons are made between each pair of methods at the $99^{\text {th }}, 95^{\text {th }}$, and $75^{\text {th }}$ percentiles. ReliefF, SURF, TuRF, and SURF\&TuRF are labeled $R, S, T$, and ST respectively. Significance is illustrated with levels of grey (i.e. light grey indicates $0.01<\mathrm{p} \leq 0.05$, dark grey indicates $0.001<\mathrm{p} \leq 0.01$, and black indicates $\mathrm{p} \leq 0.001$ ).

Click here for file

[http://www.biomedcentral.com/content/supplementary/17560381-2-5-S4.pdf]

\section{Additional file 5}

Significance of differences with a sample size of 3200. This is a plot showing the significance of statistical results for the situation where there are 1600 cases and 1600 control individuals. These plots follow the example shown in Figure 2. Pairwise comparisons are made between each pair of methods at the 99th $95^{\text {th }}$, and $75^{\text {th }}$ percentiles. ReliefF, SURF, TuRF, and SURF\&TuRF are labeled $R, S, T$, and ST respectively. Significance is illustrated with levels of grey (i.e. light grey indicates $0.01<\mathrm{p} \leq 0.05$, dark grey indicates $0.001<\mathrm{p} \leq 0.01$, and black indicates $\mathrm{p} \leq 0.001)$. Click here for file

[http://www.biomedcentral.com/content/supplementary/17560381-2-5-S5.pdf]

\section{Acknowledgements}

This work is funded by NIH grants LM0090 I2, Al59694, HD047447, and ES007373. The authors would like to thank Mr. Jason Gilmore for his technical assistance.

\section{References}

I. Iles MM: What Can Genome-Wide Association Studies Tell Us about the Genetics of Common Disease? PLoS Genet 2008, 4(2):e33.

2. McCarthy MI, Abecasis GR, Cardon LR, Goldstein DB, Little J, loannidis JPA, Hirschhorn JN: Genome-wide association studies for complex traits: consensus, uncertainty and challenges. Nat Rev Genet 2008, 9(5):356-369.

3. Hardy J, Singleton A: Genomewide Association Studies and Human Disease. N Engl J Med 2009, 360(17): 1759-1768.

4. Kraft P, Hunter DJ: Genetic Risk Prediction - Are We There Yet? N Engl J Med 2009, 360(17): I 70I-I 703.

5. Jakobsdottir J, Gorin MB, Conley YP, Ferrell RE, Weeks DE: Interpretation of Genetic Association Studies: Markers with Replicated Highly Significant Odds Ratios May Be Poor Classifiers. PLoS Genet 2009, 5(2):e 1000337.

6. Tyler AL, Asselbergs FW, Williams SM, Moore JH: Shadows of complexity: what biological networks reveal about epistasis and pleiotropy. BioEssays 2009, 3 I (2):220-227.
7. Hirschhorn JN, Lohmueller K, Byrne E, Hirschhorn K: A comprehensive review of genetic association studies. Genet Med 2002, 4:45-61.

8. Finckh U: The future of genetic association studies in Alzheimer disease. Journal of Neural Transmission 2003, I I 0(3):253-266.

9. Moore JH, Ritchie MD: The Challenges of Whole-Genome Approaches to Common Diseases. JAMA 2004, 29 I(13): I642-1643.

10. Kira K, Rendell LA: A Practical Approach to Feature Selection. Machine Learning: Proceedings of the AAAl'92 1992.

II. Moore JH, White BC: Exploiting expert knowledge in genetic programming for genome-wide genetic analysis. Lecture Notes in Computer Science 2006, 41 93:969-977.

12. Moore JH, White BC: Genome-wide genetic analysis using genetic programming: The critical need for expert knowledge. Genetic Programming Theory and Practice 2007, 4: II-28.

13. Greene CS, White BC, Moore JH: An Expert Knowledge-Guided Mutation Operator for Genome-Wide Genetic Analysis Using Genetic Programming. Lecture Notes in Bioinformatics 2007, 4774:30-40.

14. Greene CS, White BC, Moore JH: Ant Colony Optimization for Genome-Wide Genetic Analysis. Lecture Notes in Computer Science 2008, 521 7:37-47.

15. Motsinger A, Reif D, Fanelli T, Davis A, Ritchie M: Linkage Disequilibrium in Genetic Association Studies Improves the Performance of Grammatical Evolution Neural Networks. IEEE Symposium on Computational Intelligence and Bioinformatics and Computational Biology, 2007. CIBCB'07 2007:I-8.

16. Moore JH, Gilbert JC, Tsai CT, Chiang FT, Holden T, Barney N, White BC: A flexible computational framework for detecting, characterizing, and interpreting statistical patterns of epistasis in genetic studies of human disease susceptibility. Journal of Theoretical Biology 2006, 24 I (2):252-26I.

17. Greene CS, Kiralis J, Moore JH: Nature-Inspired Algorithms for the Genetic Analysis of Epistasis in Common Human Diseases: Theoretical Assessment of Wrapper vs. Filter Approaches. Proceedings of the IEEE Congress on Evolutionary Computing 2009:800-807.

18. Kononenko I: Estimating Attributes: Analysis and Extensions of RELIEF. European Conference on Machine Learning 1994: I7I-I82.

19. Moore JH, White BC: Tuning ReliefF for Genome-Wide Genetic Analysis. Lecture Notes in Computer Science 2007, 4447: $166-175$.

20. Moore JH: The Ubiquitous Nature of Epistasis in Determining Susceptibility to Common Human Diseases. Human Heredity 2003, 56:73-82.

21. McKinney B, Reif D, White B, Crowe J, Moore J: Evaporative cooling feature selection for genotypic data involving interactions. Bioinformatics 2007, 23(16):2। I3-2। 20.

22. McKinney BA, Crowe JE, Guo J, Tian D: Capturing the Spectrum of Interaction Effects in Genetic Association Studies by Simulated Evaporative Cooling Network Analysis. PLOS Genet 2009, 5(3): 1000432

23. Robnik-Sikonja M, Kononenko I: An adaptation of Relief for attribute estimation in regression. In ICML '97: Proceedings of the Fourteenth International Conference on Machine Learning San Francisco, CA, USA: Morgan Kaufmann Publishers Inc; 1997:296-304.

24. Robnik-Sikonja M, Kononenko I: Theoretical and Empirical Analysis of ReliefF and RReliefF. Mach Learn 2003, 53:23-69.

25. Beretta L, Cappiello F, Moore JH, Barili M, Greene CS, Scorza R: Ability of epistatic interactions of cytokine single-nucleotide polymorphisms to predict susceptibility to disease subsets in systemic sclerosis patients. Arthritis and Rheumatism 2008, 59(7):974-83.

26. Sokal RR, Rohlf FJ: Biometry: the principles and practice of statistics in biological research 3rd edition. New York: W. H. Freeman and Co; 1995. 